Revue d'histoire de l'Amérique française

REVUE D'HISTOIRE DE L'AMÉRIQUE FRANÇAISE

\title{
Le comportement démographique des marchands de Montréal vers 1760
}

\section{José E. Igartua}

Volume 33, numéro 3, décembre 1979

URI : https://id.erudit.org/iderudit/303795ar

DOI : https://doi.org/10.7202/303795ar

Aller au sommaire du numéro

Éditeur(s)

Institut d'histoire de l'Amérique française

ISSN

0035-2357 (imprimé)

1492-1383 (numérique)

Découvrir la revue

Citer cet article

Igartua, J. E. (1979). Le comportement démographique des marchands de Montréal vers 1760. Revue d'histoire de l'Amérique française, 33(3), 427-445. https://doi.org/10.7202/303795ar d'utilisation que vous pouvez consulter en ligne.

https://apropos.erudit.org/fr/usagers/politique-dutilisation/ 


\title{
LE COMPORTEMENT DÉMOGRAPHIQUE DES MARCHANDS DE MONTRÉAL VERS 1760*
}

\author{
JOSÉ E. IGARTUA \\ Département des sciences humaines \\ Université du Québec à Chicoutimi
}

Grâce aux données fournies par la démographie historique, l'histoire sociale s'intéresse aujourd'hui à l'étude des grands événements de la vie - naissance, mariage, conception, décès - et s'efforce de comprendre comment, à travers les siècles, les attitudes et les comportements face à ces événements ont pu évoluer. On s'interroge de plus en plus vivement sur les origines de ce qu'il est convenu d'appeler la "transition démographique", c'est-à-dire, dans le monde atlantique, le déclin d'un système démographique où la fertilité est dans l'ensemble déterminée par des événements extérieurs à la volonté du couple, et l'avènement d'un système démographique dans lequel le couple choisit délibérément de limiter le nombre de ses enfants. Cette transition démographique est pour certains le signe d'une modernisation des mentalités antérieures à la modernisation des structures économiques; pour d'autres, elle reflète le pétrissage des mentalités par l'évolution socio-économique. Cause et effet à la fois, la transition démographique n'a pas procédé partout aux mêmes rythmes ni de façon égale à travers les couches sociales ${ }^{1}$.

Pour bien saisir les rythmes de diffusion du nouveau système démographique, pour en préciser les tempos culturels et en décrire la gamme des variations socio-professionnelles, il faut procéder, autant qu'à des monographies locales ou régionales, à l'analyse des comportements démographiques des différentes couches sociales à travers le temps. Or ce genre de recherches, s'il répond à l'une des

* L'auteur tient à remercier la Faculté des sciences sociales de l'Université Western Ontario pour sa contribution financière et pour le temps d'ordinateur alloué à cette recherche. Voir Edward Shorter, Naissance de la famille moderne (Paris, Seuil, 1977) et Michael Zukerman, "Dreams that Men Dare to Dream: The Role of Ideas in Western Modernization”, Social Science History, 2 (1978): 332-345. 
principales interrogations de la démographie historique, fait appel à un éventail assez large de sources disparates, incomplètes et imprécises à partir desquelles il n'est pas facile de définir et de classer les caractéristiques socio-professionnelles de la population. L'étude différentielle des comportements démographiques en a souffert autant que les autres aspects de l'histoire sociale.

Les premiers travaux ont porté, assez naturellement, sur les couches sociales les plus visibles. On a constaté, en France comme en Angleterre, que le comportement démographique de la noblesse se distinguait clairement de celui de la masse de la population, soumise à des impératifs économiques plus lourds et immergée dans un courant idéologique différent. Certaines monographies ont indiqué des âges au mariage et des tailles de famille différentes selon les couches sociales, ce qui en a porté d'aucunsà y voir un signe de la modernisation des mentalités, et d'autres à y constater tout simplement les effets différents des milieux socio-économiques ruraux et urbains sur le comportement démographique. Il est assez difficile, par ailleurs, de pouvoir isoler l'effet de l'une ou l'autre de ces variables ${ }^{2}$.

Les études de démographie différentielle qui portent sur les populations pré-industrielles de l'Amérique du Nord sont encore rares. Les travaux les plus avancés sont sans doute ceux du Programme de Recherche en Démographie historique de l'Université de Montréal, dont la reconstitution de la population canadienne aux dix-septième et dix-huitième siècles permettra de répondre à une foule de questions, mais la constitution de cette banque de données est encore en cours ${ }^{3}$. Des recherches sur les attitudes et les comportements économiques des marchands de Montréal, au milieu du dix-huitième siècle, nous ont cependant amené à la reconstitution de leurs familles afin de saisir la nature du comportement démographique des marchands. La comparaison des données qui en ressortent avec d'autres études démographiques portant sur la Nouvelle-France, notamment la reconstitution de familles effectuée par Jacques Henripin, qui porte sur un échantillon de familles

2 Pour un résumé des acquis en démographie historique en Europe, voir André Armengaud, La famille et l'enfant en France et en Angleterre du XVIe au XVIIIe siècle (Paris, Société d'édition d'enseignement supérieur, 1975) et François Lebrun, La vie conjugale sous l'Ancien Régime (Paris, Armand Colin, 1975).

3 Pour une description de ce programme voir Jacques Légaré, André LaRose et Raymond Roy, "Reconstitution de la population canadienne au XVIle siècle: méthodes et bilan d'une recherche", Recherches sociographiques, 14 (1973): 383-400. 
canadiennes constituées entre 1700 et $1730^{4}$, permet une première évaluation du comportement démographique d'une partie de la "bourgeoisie" canadienne.

Le groupe analysé comprend les 92 familles constituées par les marchands de Montréal en affaires durant la période de la Conquête. Comme il n'existe pas de recensements de la ville de Montréal entre 1741 et 1781 , les noms des marchands ont été relevés dans les répertoires des notaires dont les greffes ont été déposés au Palais de justice du district de Montréal et qui couvrent la période de 1750 à 1775 . A été incluse dans le groupe toute personne de sexe masculin résidant à Montréal, apparaissant comme partie à au moins deux actes notariaux, l'un avant 1760 et l'autre après, et étant désignée comme "marchand", "négociant" ou "équipeur". Cette définition avait pour objet de constituer une cohorte dont il était raisonnable de penser que la profession principale était le commerce, éliminant ceux qui apparaissent trop furtivement dans les sources.

À première vue, cette manière de procéder pourrait exclure les marchands qui ne fréquentaient pas les notaires, ceux sans doute dont l'importance des affaires était la plus faible; l'étude des caractéristiques du groupe a montré que notre définition était de fait assez lâche pour retenir des marchands de tout calibre: en effet, sous le régime français, rares étaient les marchands qui pouvaient éviter d'avoir recours à un notaire soit pour une vente à crédit, soit pour une transaction immobilière, soit pour les contrats d'engagement de la main-d'oeuvre pour la traite des fourrures.

Les 92 familles comprennent 14 familles de négociants, 51 familles de marchands équipeurs, 11 familles de "marchands voyageurs", 12 familles de "marchands" sans autre précision, 3 familles de marchands-artisans et une famille dont le chef s'occupait principalement de prêter de l'argent ${ }^{5}$. Cette répartition, fondée sur l'étude des activités des marchands telles qu'elles apparaissent dans les documents notariaux (contrats de société, contrats d'engagement, contrats de vente, obligations, comptes), reproduit assez fidèlement la structure du commerce montréalais au dix-huitième siècle. Le gros du commerce portait sur les fourrures, alors qu'une

\footnotetext{
4 Jacques Henripin, La population canadienne au début du XVIIIe siècle (Paris, P.U.F., 1954).

's Sept marchands qui sont demeurés célibataires ont été naturellement exclus de l'analyse démographique.
} 
minorité aisée de négociants contrôlait l'approvisionnement montréalais en produits importés et en marchandises de traite 6 . De plus, le groupe forme une cohorte assez homogène: le marchand "moyen" serait né en 1713, se serait marié pour une première fois en 1744 et serait décédé en 1778; il aurait été en pleine carrière en 1760, conformément aux critères de sélection utilisés pour la constitution du groupe.

La reconstitution des familles de ces 92 marchands s'est faite en trois étapes. Nous avons d'abord relevé les données pertinentes comprises dans le dictionnaire généalogique de $\mathrm{Mgr}$ Tanguay? Nous avons ensuite vérifié ces données dans les registres d'état civil du district de Montréal, laissant de côté les quelques données provenant des registres d'autres juridictions. Cette vérification révéla un nombre négligeable d'erreurs de transcription commises par Tanguay. Enfin, les lacunes du dictionnaire quant aux mentions de décès des conjoints ou aux naissances d'enfants ont été comblées autant qu'il était possible en dépouillant systématiquement l'index des sépultures et l'index des baptêmes pour la paroisse Notre-Dame de Montréal. Le nombre de dates de décès connues des conjoints passa de cette façon de 17 à 50 pour les hommes et de 25 à 54 pour les femmes. Le nombre des enfants connus passa de 333 à 609. Les omissions du dictionnaire de Tanguay pour ce qui touche la fin du régime français, du moins pour Montréal, sont donc considérables, plus encore que ne l'indiquent les résultats de notre vérification, car celle-ci n'a pas cherché de façon systématique à repérer les actes consignés dans les registres des paroisses avoisinant Montréal. Il semble que Mgr Tanguay n'aie pas poursuivi systématiquement ses reconstitutions de familles pour les dernières années du régime français: en grande majorité, les naissances omises dans le dictionnaire ont eu lieu après 1750.

Les données dont nous disposons sont donc incomplètes: il manque un nombre important de dates de décès des parents et un nombre impossible à déterminer, mais sans doute relativement faible, de naissances d'enfants. De plus, nous n'avons que quelques mentions de décès des enfants; nous avons renoncé à les rechercher,

6 Voir José Igartua, "The Merchants and Negociants of Montréal, 1750-1775: A Study in Socio-Economic History" (thèse de Ph. D., Michigan State University, 1974). Nous corrigeons ici les calculs rapides, fondés uniquement sur le dictionnaire Tanguay, que nous avions rapportés dans ce travail.

7 Cyprien Tanguay, Dictionnaire généalogique des familles canadiennes (7 vols., Montréal, E. Sénécal, 1871-1890). 
la tâche étant trop considérable pour les besoins de cette étude. Néanmoins, les données recueillies permettent d'établir certaines pratiques démographiques, notamment celles qui sont pertinentes au mariage et à la conception des enfants. Ces pratiques, à leur tour, peuvent servir de révélateur des attitudes concernant la reproduction et plus généralement des attitudes devant la vie.

\section{Les mariages}

Dans les populations pré-malthusiennes, l'âge des conjoints au mariage détermine en grande mesure le nombre maximum d'enfants qu'un couple peut produire. On en a déduit que le mariage tardif serait une première manifestation de l'esprit calculateur, voire de l'esprit d'entreprise. André Burguière a posé comme hypothèse que la pratique du mariage tardif témoigne de l'avènement d'une mentalité calculatrice: "la préoccupation du couple n'est plus simplement de fabriquer une famille, mais de savoir la gérer, de préserver et d'améliorer son statut social, devenu sa principale finalité"8. Le mariage signifierait la formation d'une unité de production et de consommation et le début de la période de reproduction, la stratégie du mariage tardif répondant à des considérations d'ordre économique: le désir d'avoir les moyens jugés suffisants pour assurer l'établissement et la survie matérielle du ménage avec l'arrivée des enfants. Cette stratégie implique l'abandon d'une vision fataliste de la vie et l'espoir que l'homme puisse contrôler son destin. Burguière va jusqu'à comparer ce qu'il appelle l'"esprit d'entreprise matrimonial" à l'esprit d'entreprise capitaliste, voyant dans le premier l'“archétype" du deuxième ${ }^{9}$.

L'idée avancée par Burguière est audacieuse. Pour la vérifier, on peut en tirer le modèle suivant: plus l'esprit d'entreprise est développé dans un groupe donné, plus la stratégie du mariage tardif y sera fréquente. Une population de marchands constitue un lieu privilégié pour vérifier cette hypothèse. Il est raisonnable, en effet, de penser que c'est chez les marchands qu'un tel esprit calculateur a le plus de chances de se manifester. Dans la mesure où les âges au mariage chez les marchands sont plus élevés que ceux de l'ensemble de la population, le modèle tiré de l'hypothèse de Burguière se vérifie. Le tableau suivant permet de comparer les âges au premier mariage calculés par Jacques Henripin pour un échantillon de la

8 André Burguière, “De Malthus à Max Weber: le mariage tardif et l'esprit d'entreprise”, Annales E.S.C., 27 (1972): 1128-1138. La citation provient de la page 1138.

9 Ibid. 
population canadienne au début du dix-huitème siècle avec les chiffres portant sur des groupes plus petits: un échantillon de familles canadiennes du dix-septième siècle, la population montréalaise au dix-septième siècle, un échantillon de familles de voyageurs du dix-septième siècle, les familles des marchands de Montréal vers 1760, et enfin les familles des marchands de Buenos Aires à la fin du dix-huitième siècle, ces dernières données constituant les seuls chiffres à notre connaissance se rapportant à une catégorie socio-

Tableau 1

ÂGES MOYENS AU PREMIER MARIAGE

\begin{tabular}{lcccccc}
\hline & $\begin{array}{c}\text { Canada } \\
\text { 18e siècle }\end{array}$ & $\begin{array}{c}\text { Canada } \\
17 \text { siècle }^{2}\end{array}$ & $\begin{array}{c}\text { Montréal } \\
17 \mathrm{e}^{2} \text { siècle }^{3}\end{array}$ & $\begin{array}{c}\text { Voyageurs } \\
17 \text { s siècle }^{4}\end{array}$ & $\begin{array}{c}\text { Marchands } \\
\text { de Montréal }\end{array}$ & $\begin{array}{c}\text { Marchands } \\
\text { de Buenos Aires }\end{array}$ \\
\hline Hommes & 26,9 & 27,0 & 28,6 & 29,2 & 30,9 & 35 \\
Femmes & 22,4 & 22,0 & 21,0 & $-25,1$ & 18 \\
\hline
\end{tabular}

1 Jacques Henripin, La population canadienne au début du XVIIIe siècle (Paris, P.U.F., 1954), 96.

2 Hubert Charbonneau, Vie et mort de nos ancêtres (Montréal, Presses de l'Université de Montréal, 1975), 58.

3 Louise Dechêne, Habitants et marchands de Montréal au XVIIIe siècle (MontréalParis, Plon, 1974), 104.

4 Hubert Charbonneau, Bertrand Desjardins et Pierre Beauchamp, "Le comportement démographique des voyageurs sous le régime français”, Histoire sociale - Social History, 11 (1978): 130.

5 Susan Migden Socolow, The merchants of Buenos Aires 1778-1810 (New York, Cambridge University Press, 1978), 40.

professionnelle comparable à celle que nous avons étudiée. On remarque que si dans l'ensemble les Montréalais au dix-septième siècle se marient légèrement plus tard que la moyenne pour la colonie, les groupes spécifiquement urbains, les voyageurs et les marchands, se marient encore plus tard; ces derniers attendent quatre ans de pius que la population en général avant de former une première union. On remarque aussi qu'ils choisissent des épouses qui ont deux ans et demi de plus que l'épouse-type au dix-huitième siècle. 
La ventilation de ces données par sous-catégorie professionnelle fait ressortir des différences importantes dans l'âge au premier mariage des conjoints. Ce ne sont pas les négociants, sans doute

Tableau 2

ĀGES AU PREMIER MARIAGE PAR SOUS-CATÉGORIE PROFESSIONNELLE ${ }^{1}$

\begin{tabular}{lcc}
\hline & $\begin{array}{c}\text { Époux } \\
\text { Âge moyen }\end{array}$ & $\begin{array}{c}\text { Épouses } \\
\text { Âge moyen }\end{array}$ \\
\hline Négorie & Ágociants & \\
Équipeurs & $27,15(13)$ & $23,33(12)$ \\
Marchands-voyageurs & $31,65(48)$ & $34,02(49)$ \\
Marchands & $30,36(11)$ & $24,45(11)$ \\
Marchands-artisans & $34,09(11)$ & $32,82(11)$ \\
Prêteur & $26,33(3)$ & $20,00(3)$ \\
TOTAL & $25,00(1)$ & $36,00(1)$ \\
& $30,86(87)$ & $25,09(87)$ \\
\hline
\end{tabular}

1 Il s'agit de l'âge des conjoints au premier mariage de l'époux; dans 6 cas, l'épouse choisie était veuve, dont une en était à son troisième mariage.

ceux parmi les gens de commerce dont l'esprit de calcul était le plus développé, qui se marient le plus tard, mais les simples marchands. Ceux-ci choisissent d'ailleurs des épouses qui ont presque le même âge qu'eux. L'âge au premier mariage serait donc fonction du niveau de richesse des conjoints, si l'on peut juger de celui-ci, fort grossièrement, par la profession du mari. Une étude des antécédents familiaux des conjoints fournirait une vérification plus rigoureuse de cette conclusion. 


\section{Taille des familles}

À première vue, on pourrait croire que l'un des effets du mariage tardif serait d'abaisser la taille des familles, la période de fécondabilité des épouses étant réduite. Les calculs effectués par Hubert Charbonneau sur un échantillon de la population canadienne au dix-septième siècle montrent que la descendance complète d'un couple, c'est-à-dire le "nombre moyen d'enfants auxquels les couples donneraient finalement naissance, en l'absence de rupture d'union", diminue de 1,47 enfants lorsque l'âge au mariage de la femme passe de 20-24 ans à 25-29 ans ${ }^{10}$. Or le lien entre l'âge au mariage des épouses et la taille des familles s'avère assez faible chez les marchands.

\section{Tableau 3}

NOMBRE D'ENFANTS ISSUS DU PREMIER MARIAGE

\begin{tabular}{|c|c|c|c|c|c|c|}
\hline & \multirow[t]{2}{*}{$\begin{array}{l}\text { Canada } \\
18 \mathrm{e} \text { siècle }\end{array}$} & \multirow[t]{2}{*}{$\begin{array}{l}\text { Canada } \\
17 \mathrm{e} \text { siècle }\end{array}$} & \multirow[t]{2}{*}{$\begin{array}{l}\text { Voyageurs } \\
17 \text { e siècle }^{3}\end{array}$} & \multirow{2}{*}{$\begin{array}{l}\text { Marchands de } \\
\text { Montréal, } \\
1750-1775\end{array}$} & \multicolumn{2}{|c|}{$\begin{array}{l}\text { Marchands de } \\
\text { Buenos Aires }\end{array}$} \\
\hline & & & & & Riches & Autres \\
\hline $\begin{array}{l}\text { Familles } \\
\text { complètes }\end{array}$ & 8,39 & 6,8 & -- & 6,6 & -- & -- \\
\hline $\begin{array}{l}\text { Familles } \\
\text { incomplètes }\end{array}$ & 4,41 & 5,0 & -- & 6,1 & -- & -- \\
\hline $\begin{array}{l}\text { Familles } \\
\text { inconnues }\end{array}$ & -- & -- & -- & 5,6 & -- & -- \\
\hline TOTAL & 5,65 & 6,7 & 6,8 & 5,8 & 8,0 & 7,0 \\
\hline
\end{tabular}

Henripin, La population, 50.

Charbonneau, Vie et mort, 195-196.

3 Charbonneau, Desjardins et Beauchamp, "Le comportement démographique", 130.

4 Socolow, Buenos Aires, 47-48.

10 Hubert Charbonneau, Vie et mort de nos ancêtres (Montréal, Presses de l'Université de Montréal, 1975), 207. 
Le tableau 3 indique à première vue que les marchands de Montréal ont des familles de taille semblable à celles de la population en général, en dépit de l'âge au mariage plus élevé des épouses de marchands. Mais cette moyenne cache une forte variation de la taille des familles au sein du groupe des marchands. Comme à Buenos Aires, mais de façon beaucoup plus marquée, le groupe le

Tableau 4

NOMBRE D'ENFANTS DES FAMILLES DE MARCHANDS PAR SOUS-CATÉGORIE PROFESSIONNELLE

\begin{tabular}{lcccr}
\hline Sous-catégorie & $\begin{array}{c}\text { Familles } \\
\text { complètes }\end{array}$ & $\begin{array}{c}\text { Familles } \\
\text { incomplètes }\end{array}$ & $\begin{array}{c}\text { Familles } \\
\text { inconnues }\end{array}$ & Ensemble \\
\hline Négociants & $16,00(1)$ & $13,67(3)$ & $7,30(10)$ & $9,28(14)$ \\
Equipeurs & $9,00(3)$ & $4,31(13)$ & $5,66(35)$ & $5,51(51)$ \\
Marchands-voyageurs & $9,00(2)$ & $6,33(3)$ & $3,33(6)$ & $5,18(11)$ \\
Marchands & $1,33(3)$ & $5,00(2)$ & $5,57(7)$ & $4,42(12)$ \\
Marchands-artisans & $1,00(1)$ & $9,00(1)$ & $5,00(1)$ & $5,00(3)$ \\
Prêteur & -- & -- & $0,00(1)$ & $0,00(1)$ \\
TOTAL & $6,6(10)$ & $6,14(22)$ & $5,58(60)$ & $5,83(92)$ \\
\hline
\end{tabular}

plus prospère a les plus grosses familles, dont la taille dépasse de loin la moyenne de la colonie. Par contre, la stratégie du mariage tardif semble réussir aux "marchands": c'est parmi ce groupe que la relation entre le nombre d'enfants et l'âge au mariage des parents joue le plus fortement ${ }^{11}$.

Le mariage tardif des marchands et, surtout, des épouses qu'ils choisissent semble être la seule méthode de contrôle de la taille des familles qu'ils aient employée. Il ne semble pas que les marchands, une fois leur union constituée, aient eu recours à des méthodes de contrôle des naissances. Nous en avons trois indices: la faiblesse des

11 Pour ce groupe, les corrélations de Pearson (r), qui mesurent la relation linéaire, soit positive ou négative, entre deux séries de données, sur une échelle de $-1,0$ à 1,0 , sont de $-0,6964$ entre l'âge de la mère au mariage et le nombre d'enfants, et de $-0,4937$ entre l'âge du père au mariage et le nombre d'enfants, alors qu'elles sont de $-0,4609$ et de $-0,2636$ pour le groupe en général. 
intervalles intergénésiques, le faible nombre des couples complètement stériles et l'âge élevé des épouses à la dernière naissance. Les intervalles intergénésiques, pour l'ensemble des familles des marchands, sont généralement plus bas que ceux calculés par Henripin pour son échantillon de la population canadienne. L'intervalle intergénésique moyen est de 1,83 année, alors que pour l'échantillon d'Henripin, il est de 1,94 et que pour celui de Charbonneau, tiré de la population du dix-septième siècle, il est de 1,9712. Les deuxième et troisième enfants des marchands voyageurs arrivent plus tard que la moyenne, ceci s'expliquant probablement par l'occupation de leur père. Il serait toutefois hasardeux de tirer d'autres conclusions de cette ventilation, car le nombre de cas est trop petit. (voir le Tableau 5)

Douze des 92 premiers mariages n'ont pas produit d'enfants selon les données que nous avons recueillies. Trois de ces unions furent constituées lorque l'épouse avait dépassé le cap des 45 ans. Dans un quatrième cas, bien que la date de décès de l'épouse soit inconnue, on peut supposer que l'union fut stérile car l'épouse avait 41 ans lors du mariage. Une cinquième famille, pour laquelle on ignore la date de décès des époux, fut probablement stérile à cause de l'âge avancé de l'épouse au mariage ( 39 ans), qui permet de croire qu'il n'y a pas là de naissances non retrouvées. Une seule union connut une période assez longue de fécondabilité sans donner lieu à des naissances: il s'agit d'une famille dont l'épouse vécut 16 ans sans produire d'enfants. Deux ans après le décès de sa première femme, le mari prit une seconde épouse, dont il eut quatre enfants.

Les informations manquent dans les six autres cas pour établir avec précision la longueur de la période de fécondabilité infertile, ou la période pendant laquelle une épouse, en état d'avoir des enfants, n'en a pas, car nous ne connaissons pas la date de décès de l'un des conjoints. Cependant, dans quatre de ces cas, nous savons que l'épouse décède la première avant d'atteindre 45 ans et que l'époux prend une seconde femme: deux de ces secondes unions sont fertiles. La stérilité complète des couples en âge de procréer est donc un phénomène limité à six familles au minimum, soit les quatre familles dont il vient d'être question et les deux familles complètes dont ila été fait état auparavant; au maximum, il y eut neuf familles infertiles, en ajoutant celles dont il nanque la date de décès de l'un des conjoints. Le pourcentage varierait donc entre $6,5 \%$ et $9,8 \%$.

12 Charbonneau, Vie et mort, 210. 
Tableau 5

INTERVALLES INTERGÉNÉSIQUES, EN MOIS

FAMILLES DE MARCHANDS

ET POPULATION CANADIENNE

\begin{tabular}{llllllll}
\hline $\begin{array}{l}\text { Rang de } \\
\text { l'intervalle }\end{array}$ & Négociants & Equipeurs & $\begin{array}{c}\text { Marchands } \\
\text { voyageurs }\end{array}$ & Marchands & $\begin{array}{c}\text { Marchands } \\
\text { artisans }\end{array}$ & $\begin{array}{c}\text { Ensemble des } \\
\text { marchands }\end{array}$ & $\begin{array}{c}\text { Population } \\
\text { canadienne }\end{array}$ \\
\hline 1 & $21,11(9)$ & $23,22(32)$ & $34,12(8)$ & $15,67(6)$ & $15.00(1)$ & $23,48(56)$ & $22,53(154)$ \\
2 & $14,50(8)$ & $23,04(27)$ & $26,87(8)$ & $15,75(4)$ & $16,50(2)$ & $21,41(49)$ & $21,07(143)$ \\
3 & $17,75(8)$ & $22,14(21)$ & $15,50(6)$ & $24,50(4)$ & $17,50(2)$ & $20,32(41)$ & $22,63(135)$ \\
4 & $15,62(8)$ & $24,95(20)$ & $19,00(4)$ & $25,50(4)$ & $12,00(2)$ & $21,74(38)$ & $22,90(117)$ \\
5 & $16,62(8)$ & $25,79(19)$ & $20,00(3)$ & $20,33(3)$ & $12,00(1)$ & $22,34(34)$ & $25,28(101)$ \\
6 & $28,57(7)$ & $21,71(14)$ & $21,00(3)$ & $29,67(3)$ & $14,00(1)$ & $23,93(28)$ & $23,80(91)$ \\
7 & $19,50(8)$ & $26,70(10)$ & $20,33(3)$ & $12,00(1)$ & $21,00(1)$ & $22,48(23)$ & $25,15(73)$ \\
8 & $14,40(5)$ & $16,67(9)$ & $12,00(1)$ & -- & $16,00(1)$ & $15,62(16)$ & $25,96(54)$ \\
9 & $20,20(5)$ & $18,75(8)$ & $13,00(1)$ & -- & -- & $18,86(14)$ & $24,60(43)$ \\
10 & $18,20(5)$ & $17,50(6)$ & $17,00(1)$ & -- & -- & $17,75(12)$ & $24,65(34)$ \\
11 & $25,00(4)$ & $23,00(5)$ & $54,00(1)$ & -- & -- & $26,90(10)$ & $23,10(24)$ \\
12 & $18,25(4)$ & $27,50(4)$ & $18,00(1)$ & -- & $-\ldots$ & $22,33(9)$ & $21,57(16)$ \\
\hline
\end{tabular}

1 Henripin, La population canadienne, 85. 
Si les cas de stérilité complète sont rares, d'autres facteurs entrent en jeu pour réduire la taille moyenne des familles sans que la volonté des conjoints y soit pour quelque chose. Le facteur le plus évident est la mort de l'un des conjoints avant que ne se termine la période de fertilité du couple. Pour les familles de marchands en général, l'âge moyen de l'épouse à la naissance du dernier enfant se situe à 35 ans, ce qui reflète le poids des familles incomplètes (où l'union se termine par le décès de l'un des conjoints avant la fin de la période de fertilité de l'épouse) et des familles inconnues (dont il manque la date de décès de l'un ou l'autre des conjoints) dans la population étudiée. Le groupe ne comprend que 10 familles complètes et 22 familles incomplètes, le reste comptant parmi les familles inconnues. Parmi les familles complètes, l'âge moyen de l'épouse à la naissance du dernier enfant est de 40 ans, alors que chez les familles incomplètes la moyenne est de 35 ans comme pour tout le groupe, les familles inconnues comprises. Les moyennes calculées pour l'échantillon d'Henripin se situent à 41 ans pour les familles complètes et à 31 ans pour les familles incomplètes ${ }^{13}$. La différence entre les deux groupes de familles incomplètes peut être attribuable à plusieurs facteurs, dont des différences de mortalité entre les deux groupes, qui pourraient s'expliquer par des différences de niveau de vie ou des différences dans la répartition par groupe d'âge des épouses, ou simplement par la faible taille de notre groupe et la forte dispersion des données (de 22 ans à 48 ans pour 20 cas seulement). Cependant, avec les indications précédentes, ces chiffres tendent à confirmer l'absence de limitation des naissances chez les marchands comme dans la population en général.

Avant de clore la discussion sur la taille des familles, il convient de remarquer le faible taux de masculinité des enfants des marchands, $94,5 \%$ pour les enfants du premier lit et $124,24 \%$ pour les enfants du deuxième lit, pour une moyenne de $97,73 \%$, alors qu'Henripin trouve un taux de $103,2 \%{ }^{14}$. On pourrait se demander si la taille de certaines familles ne serait pas influencée par le désir de produire un héritier mâle: il faudrait ici les dates de décès des enfants pour établir la taille de la famille et la proportion de mâles parmi les enfants encore vivants au moment d'une conception. Nous n'avons pas pu faire cette analyse faute de données, mais la ventilation des taux de masculinité comme l'examen des couples de négociants, qui

13 Henripin, La population, 54.
14 Ibid. 
sont les plus féconds, nous inclinent à rejeter une telle interprétation: le taux de masculinité ne varie pas avec la taille des familles et chacune des quatorze familles de négociants produit au moins un enfant mâle parmi les trois premières naissances.

Le nombre des conceptions illégitimes - les enfants nés avant le huitième mois du premier mariage - est de 5 parmi 535 conceptions, soit 9,34 pour 1,000 , ce qui est inférieur aux chiffres de 11,71 et de 12,21 compilés par Mgr Tanguay pour les périodes 1741-1750 et 1751-1760. Ce taux constitue cependant une mesure discutable puisqu'il reflète à la fois les variations dans la taille des familles et le nombre de conceptions illégitimes. Une mesure plus juste, ici, est le nombre de premières conceptions qui eurent lieu avant le mariage. Sur les 60 familles pour lesquelles les données nécessaires sont disponibles, 4 couples ont eu un enfant moins de six mois après leur mariage, soit $6,67 \%$ contre $4,55 \%$ dans l'échantillon d'Henripin ${ }^{15}$. Peut-on tirer des conclusions sur la vertu des marchands et de leurs épouses à partir de chiffres si petits? Il serait difficile d'y voir une différence marquée entre les marchands et la population en général.

Enfin, notons une paire de jumeaux parmi les enfants du premier lit.

\section{Variations saisonnières et quotidiennes}

Dans les sociétés d'ancien régime, le comportement démographique des couples variait considérablement selon les saisons: l'influence de l'Eglise et le rythme saisonnier du travail et de la maladie répartissaient inégalement les mariages, les naissances et les décès. Ces variations saisonnières sont particulièrement marquées pour les mariages. Les données dont nous disposons pour le Canada aux dix-septième et dix-huitième siècles concordent assez bien entre elles: aux fréquences assez fortes de janvier et de février succède une période creuse au mois de mars, temps de Carême où l'Église prohibe les mariages, puis une reprise en avril et une longue période calme jusqu'en septembre, les mois d'octobre et de novembre constituant une saison forte, et décembre, mois de l'Avent, fermant l'année par une autre période creuse (voir le Tableau 6).

$15 \quad$ Ibid., 55. 


\section{Tableau 6}

\section{MOUVEMENT SAISONNIER DES MARIAGES}

\begin{tabular}{|c|c|c|c|c|c|c|c|c|}
\hline \multirow[b]{2}{*}{$\begin{array}{l}\text { Mois du } \\
\text { mariage }\end{array}$} & \multicolumn{2}{|c|}{ Henripin 1} & \multicolumn{2}{|c|}{ Dechêne $^{2}$} & \multicolumn{2}{|c|}{ Charbonneau ${ }^{3}$} & \multicolumn{2}{|c|}{ Igartua 4} \\
\hline & $\begin{array}{c}\text { Nombre } \\
\text { absolu }\end{array}$ & $\begin{array}{l}\text { Nombre } \\
\text { relatif* }\end{array}$ & $\begin{array}{c}\text { Nombre } \\
\text { absolu }\end{array}$ & $\begin{array}{c}\text { Nombre } \\
\text { relatif }\end{array}$ & $\begin{array}{c}\text { Nombre } \\
\text { absolu }\end{array}$ & $\begin{array}{c}\text { Nombre } \\
\text { relatif }\end{array}$ & $\begin{array}{c}\text { Nombre } \\
\text { absolu }\end{array}$ & $\begin{array}{c}\text { Nombre } \\
\text { relatif }\end{array}$ \\
\hline Janvier & 136 & 164,88 & 148 & 132 & 415 & 133 & 18 & 214 \\
\hline Février & 139 & 186,55 & 120 & 121 & 380 & 134 & 9 & 118 \\
\hline Mars & 16 & 19,39 & 38 & 33 & 75 & 24 & 2 & 23 \\
\hline Avril & 77 & 96,49 & 80 & 74 & 241 & 80 & 10 & 123 \\
\hline Mai & 59 & 71,52 & 67 & 59 & 129 & 41 & 12 & 142 \\
\hline Juin & 66 & 82,70 & 80 & 73 & 159 & 53 & 3 & 37 \\
\hline Juillet & 64 & 77,59 & 65 & 57 & 184 & 59 & 3 & 35 \\
\hline Aout & 57 & 69,10 & 76 & 73 & 221 & 71 & 6 & 71 \\
\hline Septembre & 49 & 61,40 & 100 & 92 & 306 & 101 & 11 & 135 \\
\hline Ovtobre & 73 & 88,50 & 141 & 126 & 589 & 189 & 5 & 59 \\
\hline Novembre & 206 & 258,14 & 310 & 290 & 857 & 284 & 16 & 196 \\
\hline Décembre & 29 & 35,15 & 77 & 68 & 96 & 31 & 4 & 47 \\
\hline TOTAL & 971 & & 1302 & & 3652 & & 99 & \\
\hline
\end{tabular}

* En l'absence de variations saisonnières et de l'inégalité des mois, il y aurait 100 mariages chaque mois pour 1200 mariages célébrés durant l'année.

1 Henripin, La population canadienne, 45. Le nombre relatif a été recalculé sans tenir compte de l'influence de la tendance, afin d'être comparable aux autres nombres relatifs.

2 Dechêne, Habitants et marchands, 110.

3 Raymond Roy, Yves Landry et Hubert Charbonneau, "Quelques comportements des Canadiens au XVIIe siècle d'après les registres paroissiaux", Revue d'histoire de l'Amérique française, 31, 1 (juin 1977): 54.

4 Comprend les remariages.

Le mouvement saisonnier des mariages chez les marchands de Montréal suit assez bien ce mouvement mais il exhibe aussi des divergences: la force plus grande des mois de janvier, avril, mai et septembre, particulièrement celle du mois de mai, et la plus grande faiblesse du mois d'octobre. Ces divergences sont difficiles à expliquer. Les périodes creuses de juin et juillet, mois d'intense activité commerciale, et d'octobre, saison de départ des navires pour la France, emportant avec eux lettres de change et commandes pour l'année suivante, forçaient sans doute les marchands à reporter leurs 
mariages sur les mois fastes de l'année. On saisit mal, cependant, la préférence relativement plus marquée pour les mois de janvier et mai que pour le mois de novembre. Une étude sur les marchands de Québec pourrait sans doute nous apporter des éléments de comparaison intéressants.

Les fluctuations des fréquences des naissances sont moins grandes que pour les mariages mais ici encore ces fluctuations sont plus violentes dans le cas des marchands que pour les autres groupes. Ces fluctuations reflètent en partie l'influence des premières naissances, particulièrement aux mois de janvier, février, avril, septembre et décembre, mais ce facteur n'influence que légèrement la courbe générale. Par rapport aux autres mouvements des naissances, la courbe des marchands exhibe une faiblesse plus accentuée des naissances de février, avril et août, et donc des conceptions de mai, juillet et novembre, mois de travail et de déplacements, ce qui explique sans doute l'amplitude des conceptions des mois suivants, juin, août et décembre (les naissances de mars, mai et septembre (voir le Tableau 7).

Enfin, la répartition mensuelle des décès des marchands et de leurs premières épouses se distingue nettement de celle de la population canadienne du dix-septième siècle examinée par Roy, Landry et Charbonneau, qui comprend les décès des enfants comme ceux des adultes. La fin de l'hiver et le début du printemps marquent les temps les plus difficiles: on pense à la dureté du climat et à l'appauvrissement de la diète durant cette saison comme causes évidentes, mais qui ne rendent pas compte de la forte poussée des décès en octobre (voir le Tableau 8).

Les variations quotidiennes des mariages, des baptêmes et des sépultures révèlent certaines autres différences entre la population du dix-septième siècle et le groupe des marchands de la période de la Conquête. Les variations dans les fréquences quotidiennes des mariages sont à peu près les mêmes dans les deux cas: prépondérance des premiers jours de la semaine, les lundis et mardis, dans la célébration du mariage (voir le Tableau 9).

Mais la répartition quotidienne des baptêmes diffère légèrement d'un groupe à l'autre. Les marchands respectaient scrupuleusement les ordonnances de l'Église concernant le baptême le plus tôt possible après la naissance: $64,9 \%$ des enfants des marchands (premier et deuxième lits) furent baptisés le jour de leur naissance et 
Tableau 7

MOUVEMENT SAISONNIER DES NAISSANCES

\begin{tabular}{lcccccccc}
\hline & \multicolumn{2}{c}{ Henripin $^{1}$} & \multicolumn{2}{c}{ Dechêne $^{2}$} & \multicolumn{2}{c}{ Charbonneau $^{3}$} & \multicolumn{2}{c}{ Igartua } \\
$\begin{array}{l}\text { Mois de } \\
\text { la naissance }\end{array}$ & $\begin{array}{c}\text { Nombre } \\
\text { absolu }\end{array}$ & $\begin{array}{c}\text { Nombre } \\
\text { relatif* }\end{array}$ & $\begin{array}{c}\text { Nombre } \\
\text { absolu }\end{array}$ & $\begin{array}{c}\text { Nombre } \\
\text { relatif }\end{array}$ & $\begin{array}{c}\text { Nombre } \\
\text { absolu }\end{array}$ & $\begin{array}{c}\text { Nombre } \\
\text { relatif }\end{array}$ & $\begin{array}{c}\text { Nombre } \\
\text { absolu }\end{array}$ & $\begin{array}{c}\text { Nombre } \\
\text { relatif }\end{array}$ \\
\hline Janvier & 497 & 94,79 & 590 & 104 & 1609 & 109 & 48 & 114 \\
Février & 548 & 114,48 & 628 & 125 & 1621 & 120 & 38 & 100 \\
Mars & 691 & 130,00 & 648 & 113 & 1607 & 109 & 45 & 107 \\
Avril & 556 & 108,45 & 559 & 104 & 1461 & 102 & 32 & 78 \\
Mai & 566 & 106,81 & 541 & 94 & 1312 & 89 & 42 & 100 \\
Juin & 472 & 92,06 & 505 & 91 & 1174 & 82 & 33 & 81 \\
Juillet & 506 & 95,48 & 467 & 81 & 1185 & 80 & 34 & 80 \\
Août & 507 & 95,67 & 569 & 99 & 1491 & 101 & 34 & 80 \\
Septembre & 494 & 96,36 & 597 & 107 & 1553 & 109 & 59 & 144 \\
Octobre & 550 & 103,78 & 581 & 103 & 1516 & 103 & 51 & 121 \\
Novembre & 475 & 92,65 & 501 & 91 & 1387 & 97 & 42 & 103 \\
Décembre & 376 & 70,95 & 498 & 86 & 1456 & 99 & 39 & 92 \\
TOTAL & 6238 & & 6684 & & 17372 & & 497 &
\end{tabular}

* En l'absence de variations saisonnières et de l'inégalité des mois, il y aurait 100 naissances chaque mois pour 1200 naissances durant l'année.

Henripin, La population canadienne, 42,45 . Somme des premières naissances et des naissances ultérieures, et calcul du nombre relatif en ne tenant pas compte de l'influence de la tendance.

2 Dechêne, Habitants et marchands, 113.

3 Roy, Landry et Charbonneau, "Quelques comportements", 61. Il s'agit ici de baptêmes. 


\section{Tableau 8}

MOUVEMENT SAISONNIER DES DÉCÈS

\begin{tabular}{lcccc}
\hline $\begin{array}{l}\text { Mois du } \\
\text { décès }\end{array}$ & Marchands & $\begin{array}{c}\text { Premières } \\
\text { épouses }\end{array}$ & Total & $\begin{array}{c}\text { Nombre } \\
\text { relatif* }\end{array}$ \\
\hline Janvier & 5 & 2 & 7 & 91 \\
Février & 4 & 5 & 9 & 130 \\
Mars & 7 & 4 & 11 & 143 \\
Avril & 4 & 6 & 10 & 138 \\
Mai & 3 & 8 & 11 & 143 \\
Juin & 2 & 2 & 4 & 55 \\
Juillet & - & 1 & 1 & 13 \\
Août & 3 & 3 & 6 & 78 \\
Septembre & 1 & 2 & 3 & 41 \\
Octobre & 6 & 8 & 14 & 183 \\
Novembre & 1 & 3 & 4 & 55 \\
Décembre & 4 & 6 & 10 & 130 \\
TOTAL & 40 & 50 & 90 & 1200 \\
\hline
\end{tabular}

* En l'absence de variations saisonnières et de l'inégalité des mois, il y aurait 100 décès chaque mois pour 1200 décès par année.

$34,7 \%$ le lendemain. Seulement 2 baptêmes sur les 493 cas pour lesquels nous possédons les dates de naissance et de baptême eurent lieu deux jours après la naissance et aucun n'eut lieu plus longtemps après. Il s'ensuit que le mouvement quotidien des baptêmes, dans ies familles de marchands, est presque identique à celui des naissances: la répartition est à peu près uniforme et il n'apparaît pas de préférence, même légère, pour le dimanche comme c'est le cas pour la population canadienne au dix-septième siècle. Quant aux décès des marchands et de leurs premières épouses, la répartition quotidienne est elle aussi uniforme, mais celle des sépultures varie plus sensiblement, les lundis et les samedis apparaissant plus fréquemment. L'écart n'est cependant pas très grand; seulement deux sépultures sur quatre-vingt-dix surviennent plus de deux jours après le décès.

Cette première analyse démographique d'une catégorie socioprofessionnelle de la population canadienne au dix-huitième siècle ne nous permet donc pas d'apercevoir une originalité prononcée dans le comportement démographique des marchands de Montréal. Â presque tous les points de vue qu'il était possible d'examiner à 
Tableau 9

FRẼQUENCE QUOTIDIENNE DES MARIAGES, DES NAISSANCES, DES BAPTÊMES, DES DÊCES ET DES SÉPULTURES, EN NOMBRE RELATIFS, MARCHANDS DE MONTRÉAL ET POPULATION CANADIENNE AU DIX-SEPTIËME SIËCLE

\begin{tabular}{|c|c|c|c|c|c|c|c|c|}
\hline \multirow[t]{2}{*}{ Jour } & \multicolumn{2}{|c|}{ Mariages } & \multirow{2}{*}{$\begin{array}{c}\text { Naissances } \\
\text { Montréal }\end{array}$} & \multicolumn{2}{|c|}{ Baptêmes } & \multirow{2}{*}{$\begin{array}{c}\text { Décès } \\
\text { Montréal }\end{array}$} & \multicolumn{2}{|c|}{ Sépultures } \\
\hline & Montréal & Canada & & Montréal & Canada & & Montréal & Canada \\
\hline Dimanche & 7,3 & 7,3 & 15,3 & 14,6 & 20,8 & 12,2 & 15,6 & 16,7 \\
\hline Lundi & 48,3 & 46,1 & 15,1 & 15,0 & 14,3 & 13,3 & 20,8 & 14,1 \\
\hline Mardi & 16,7 & 20,7 & 14,1 & 14,8 & 13,3 & 12,2 & 7,3 & 13,4 \\
\hline Mercredi & 14,0 & 9,8 & 15,5 & 16,0 & 13,3 & 16,8 & 13,5 & 14,0 \\
\hline Jeudi & 7,9 & 9,7 & 13,7 & 14,1 & 13,4 & 12,2 & 14,6 & 13,1 \\
\hline Vendredi & 1,7 & 1,9 & 14,3 & 13,4 & 12,4 & 13,3 & 9,4 & 14,4 \\
\hline Samedi & 7,0 & 4,5 & 12,0 & 12,1 & 12,5 & 20,0 & 18,8 & 14,4 \\
\hline TOTAL & 100,0 & 100,0 & 100,0 & 100,0 & 100,0 & 100,0 & 100,0 & 100,0 \\
\hline
\end{tabular}

1 Raymond Roy, Yves Landry et Hubert Charbonneau, "Quelques comportements des Canadiens au XVIIe siècle d'après les registres paroissiaux", RHAF, 31, 1 (juin 1977): 63. 
Figure 1

\section{VARIATION MENSUELLE DES NAISSANCES ENFANTS DE MARCHANDS}

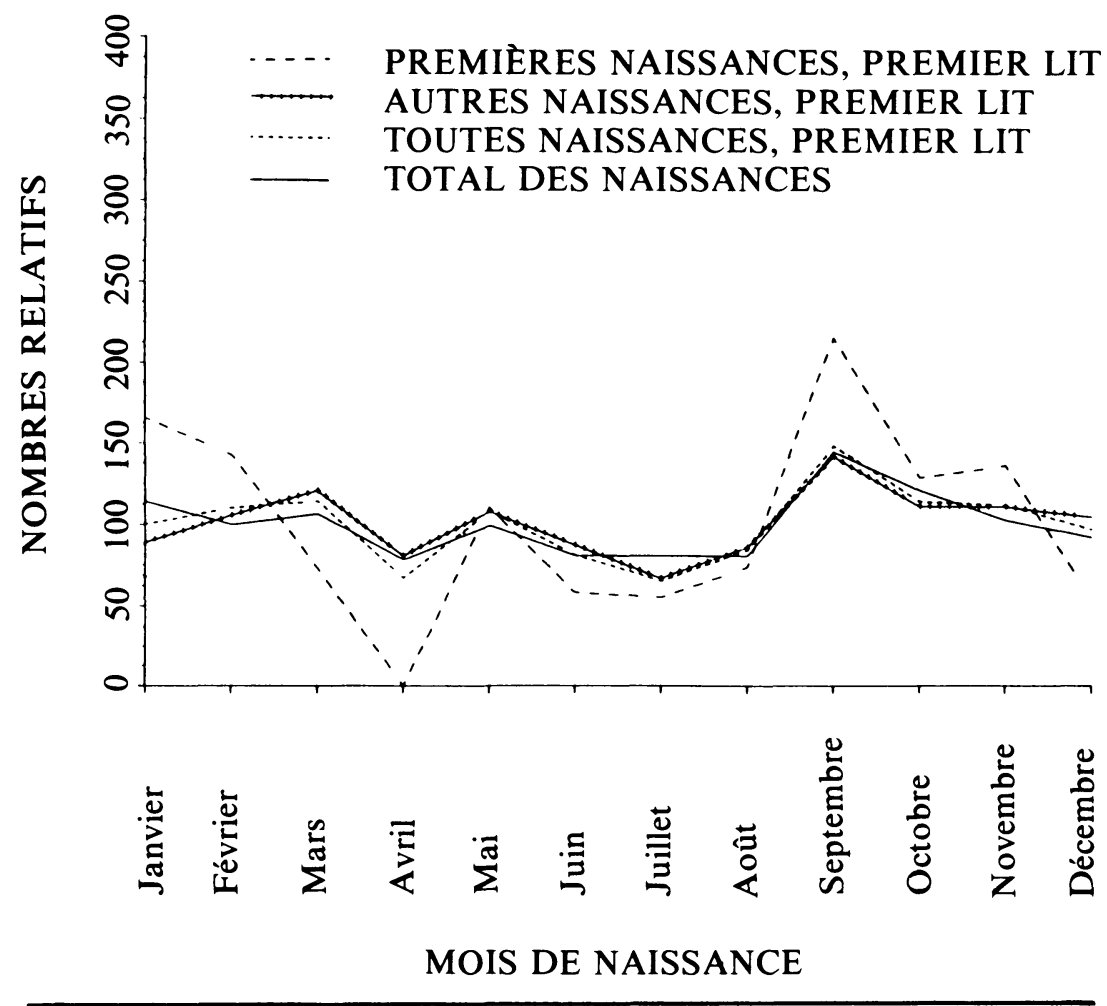

partir de nos données, ils se montrent semblables à leurs voisins d'occupation plus modeste. Seul les distingue leur âge plus avancé au mariage, ainsi que celui de leurs compagnes. Il n'est pas possible, dans l'état actuel des recherches, de dire s'ils manifestaient là des comportements semblables ou différents de ceux des autres groupes de bourgeois des colonies nord-américaines au dix-huitième siècle. Il faudrait répondre à cette question avant de juger de la mentalité des marchands par cet aspect de leur comportement. 